\title{
Study on Assessment Model of Potential Risk in Car
}

\section{Following Scenario}

\author{
Haozhou Wei, Zhixiong Ma, Xichan Zhu and Jingwei He \\ School of Automotive Studies, Tongii University, 4800 Cao'an Highway, Shanghai 201804, P. R. China
}

\begin{abstract}
Car following is the most common driving scenario, and rear-end collisions in car following scenario are the most common accidents. TTC (time to collision) is widely employed as the risk indicator in existing rear-end collision warning systems, however, assessment model using only TTC may ignore some high-risk scenarios. Therefore, the concept of potential risk in car following scenario is proposed, and the driver's maximum brake response time to avoid collision is defined as TM (time margin), which is the basis of the assessment model in this study. One hundred and thirty-nine (139) dangerous car following cases from China-FOT (Field Operation Test) naturalistic driving study database are extracted in reference from detection criteria on dangerous cases, of which TM at the braking moment of preceding vehicle is calculated, thus dangerous domains of two risk levels are determined by the distribution of TM. A potential risk warning system is also designed based on the assessment model, in order to further reduce rear-end collisions in combination with rear-end collision warning system.
\end{abstract}

Key words: Risk assessment, ADAS, naturalistic driving study, car following.

\section{Introduction}

Car following scenario is the most common scenario in naturalistic driving, and unreasonable following strategy may lead to rear-end collisions, which are the most frequent type of accidents in both China and United States according to statistics [1,2]. At present, TTC (time to collision) is widely used risk assessment indicator of car following scenario for ADAS (advanced driver assistance system) control strategy and dangerous cases detection, however, some dangerous cases may not be recognized by TTC alone, for example, when the relative velocity is close to 0 , the calculated TTC value may be extremely large or even infinite even with small range. Another parameter THW (time headway) could cover the shortage of car following risk assessment model using only TTC [3], nevertheless, velocity of the preceding vehicle is not considered in this kind of risk assessment model.

Therefore, in comparison with conventional method

Corresponding author: Ma Zhixiong, doctor, lecturer, research fields: active and passive safety. using TTC/THW, this paper defines TM (time margin) to build assessment model of potential risk in car following scenario, aiming to ensure enough responsetime for the driver to brake for collision avoidance.

\section{Materials and Method}

The data in this research are obtained from the database covering 780 dangerous cases extracted from China-FOT, in which naturalistic driving data including drivers' behavior, vehicle dynamic parameters and traffic environment information of about 130 million meters were collected by CAN (controller area network) bus, acceleration sensor and 4 video cameras. Multi-layer detection criteria for dangerous cases relating to vehicle velocity were developed based on the relationship between critical vehicle dynamic parameters, features of driver's brake pressure characteristics and TTC [4].

In comparison with the risk measured by TTC, potential risk in car following scenario suggests the risk severity supposing that the preceding vehicle rapidly decelerates. When the potential risk is high, if 
the front vehicle brakes, the risk level would rapidly rise to dangerous domain. The maximum brake response time for the driver to avoid rear-end collision is defined as TM to assess the potential risk, using velocity of ego-vehicle $\left(V_{f}\right)$ and preceding vehicle $\left(V_{p}\right)$ and relative range $(D)$ as in Eq. (1). The maximum decelerations $a_{f}$ and $a_{p}$ are supposed to be $0.75 \mathrm{~g}$ [5], and the response time of braking system $\tau$ is determined as $0.15 \mathrm{~s}$ according to Ref. [6]. In this paper, $V_{f}$ is obtained from CAN bus directly, while $V_{p}$ and $D$ are obtained by video processing using Kalman filter optimization [7].

$$
T M=\frac{D+V_{f}^{2} / 2 a_{f}-V_{p}^{2} / 2 a_{p}}{V_{f}}-\tau=\frac{D+V_{f}^{2} / 1.5 g-V_{p}^{2} / 1.5 g}{V_{f}}-0.15=T H W-0.15-\frac{\left(V_{f}+V_{p}\right)\left(V_{f}-V_{p}\right)}{1.5 g \cdot V_{f}}
$$

\section{Results}

$\mathrm{TM}$ at the braking moment of preceding vehicle of 139 dangerous car following cases is obtained, in which the preceding car decelerated and rear-end collision could happen. The distribution is shown in Fig. 1, and yellow "•" marks represent 114 light dangerous cases in this study, while red “*” marks represent 25 severe dangerous cases. The division boundaries relating to vehicle velocity for dangerous domain of each risk level are listed in Table 1.

\section{Application}

Employing the potential risk assessment model in car following scenario, as shown in Table 2, a warning system displaying the real-time potential risk is designed through HMI (human-machine interface), in order to guide the drivers to adjust their velocity appropriately working with rear-end collision warning system cooperatively.

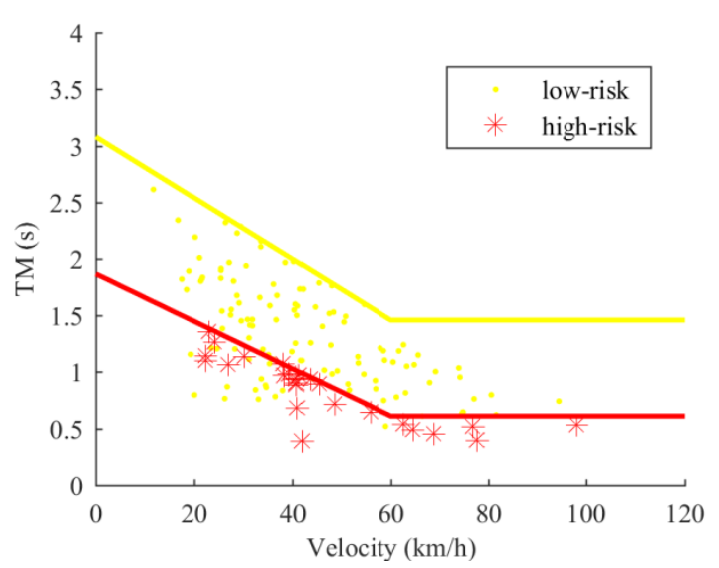

Fig. 1 Distribution of TM for different risk levels.

Table 1 Classification criteria based on TM.

\begin{tabular}{lll}
\hline Velocity $(\mathrm{km} / \mathrm{h})$ risk level & $0 \leq v<60$ & $v \geq 60$ \\
\hline Low-risk & $\leq 3.08-0.027 v$ & $\leq 1.46$ \\
High-risk & $\leq 1.87-0.021 v$ & $\leq 0.61$ \\
\hline
\end{tabular}

Table 2 Design of potential risk warning system.

\begin{tabular}{lllllll}
\hline Risk level & Velocity $(\mathrm{km} / \mathrm{h})$ & $\mathrm{TM}(\mathrm{s})$ & Display & Risk level & Velocity $(\mathrm{km} / \mathrm{h})$ & $\mathrm{TM}(\mathrm{s})$ \\
\hline \multirow{2}{*}{ Safe } & $0 \leq v<60$ & $>3.08-0.027 v$ & $>1.46$ & & $0 \leq v<60$ & $\leq 1.87-0.021 v$ \\
& $v \geq 60$ & $\leq 3.08-0.027 v$ & High-risk & $v \geq 60$ & $\leq 0.61$ \\
Low-risk & $0 \leq v<60$ & $\leq 1.46$ & & Same as rear-end collision warning \\
& $v \geq 60$ & system
\end{tabular}

\section{Conclusion}

Different from risk level suggested by TTC, this paper proposes the concept of potential risk in car following scenario, which represents the risk supposing that preceding vehicle decelerates. A new parameter TM is created to evaluate the potential risk and employed to establish the assessment model. One hundred and thirty-nine (139) dangerous car following cases are extracted from China-FOT, and the data of ego-vehicle are obtained from CAN bus directly and 
those of preceding vehicle by video processing. According to the distribution of TM at the braking moment of front vehicle, the dangerous domains of two levels are divided as shown in Table 1, and a warning system in Table 2 is designed to guide the drivers to keep the potential risk within a reasonable range and effectively reduce rear-end collisions.

\section{References}

[1] National Highway Traffic Safety Administration. "Traffic Safety Facts 2015." Accessed February 18, 2018. https://crashstats.nhtsa.dot.gov/Api/Public/ViewPublicati on $/ 812384$.

[2] Lin, Q., and Cheng, B. 2011. "Model of Driver Risk Perception in Car-Following Based on Logistic
Regression." China Journal of Highway and Transport 24 (6): 103-8.

[3] Wang, J., Xiao, Q., Chi, R., and Li, K. 2012. "Study on Vehicle forward Collision Warning Algoritnm Considering Driver Behaviotr Characteristcs on Curvy Roads."In Proceedings of the Annual Conference of ITS China, 894-904.

[4] Sun, X. 2017. "Research on the Causation and Incidents Based on Naturalistic Driving Study." Tongji University.

[5] Vangi, D., and Virga, A. 2005. "Evaluation of Emergency Braking Deceleration for Accident Reconstruction." Vehicle System Dynamics 40 (10): 895-910

[6] Sokolovskij, E. 2005. "Experimental Investigation of the Braking Process of Automobiles." Transport 20 (3): 91-5.

[7] Meng, L. 2017. "Research on Moving Status Estimation of Target Vehicle in Naturalistic Driving Data." Tongji University. 